\title{
Time-Frequency-Wavenumber Analysis of Surface Waves Using the Continuous Wavelet Transform
}

\section{Journal Article}

\section{Author(s):}

Poggi, Valerio; Fäh, Donat; Giardini, Domenico

Publication date:

2013-03

Permanent link:

https://doi.org/10.3929/ethz-b-000061301

Rights / license:

In Copyright - Non-Commercial Use Permitted

Originally published in:

Pure and Applied Geophysics 170(3), https://doi.org/10.1007/s00024-012-0505-5 


\title{
Time-Frequency-Wavenumber Analysis of Surface Waves Using the Continuous Wavelet Transform
}

\author{
V. PogGI, ${ }^{1}$ D. FÄH, ${ }^{1}$ and D. GiardinI ${ }^{1}$
}

\begin{abstract}
A modified approach to surface wave dispersion analysis using active sources is proposed. The method is based on continuous recordings, and uses the continuous wavelet transform to analyze the phase velocity dispersion of surface waves. This gives the possibility to accurately localize the phase information in time, and to isolate the most significant contribution of the surface waves. To extract the dispersion information, then, a hybrid technique is applied to the narrowband filtered seismic recordings. The technique combines the flexibility of the slant stack method in identifying waves that propagate in space and time, with the resolution of $f-k$ approaches. This is particularly beneficial for higher mode identification in cases of high noise levels. To process the continuous wavelet transform, a new mother wavelet is presented and compared to the classical and widely used Morlet type. The proposed wavelet is obtained from a raised-cosine envelope function (Hanning type). The proposed approach is particularly suitable when using continuous recordings (e.g., from seismological-like equipment) since it does not require any hardware-based source triggering. This can be subsequently done with the proposed method. Estimation of the surface wave phase delay is performed in the frequency domain by means of a covariance matrix averaging procedure over successive wave field excitations. Thus, no record stacking is necessary in the time domain and a large number of consecutive shots can be used. This leads to a certain simplification of the field procedures. To demonstrate the effectiveness of the method, we tested it on synthetics as well on real field data. For the real case we also combine dispersion curves from ambient vibrations and active measurements.
\end{abstract}

\section{Introduction}

Surface wave analysis can be done with various techniques, applicable at different scales, spanning from global tomography (e.g., SHAPIRO et al., 2005) to geotechnical site characterization (e.g., Sтокое and NAZARIAn, 1985; Tokimatsu, 1997). All of these may differ in the type of employed source (artificial or

1 Swiss Seismological Service, ETH Zurich, Sonnegstrasse 5, Zurich, Switzerland. E-mail: poggi@ sed.ethz.ch natural), frequency range (thus depth of resolution), number of receivers and analyzed component of motion. However, they all basically rely on the comparison between synchronous recordings to identify and extract the phase or group velocity dispersion characteristics of the surface waves. This information can be used to invert for the velocity structure of the site (e.g., XIA et al., 1999). If compared to other seismic methods, like reflection and refraction surveying, the increasing popularity of these techniques can be addressed to their simplicity, reliability and low cost of implementation. Despite the substantially low resolution on geometrical discontinuities (the layers interfaces), surface wave inversion is capable of robust estimation of the average seismic velocities, especially for shear waves (WATHelet et al., 2008). This is of primary importance in geotechnical engineering and local seismic hazard assessment.

Nowadays, several approaches are available to analyze the surface wave dispersion from simultaneous recordings. Most of them belong to the so-called domain-transformation methods and can be divided in two main categories: the $\tau-p$ (e.g., McMechan and YedLIN, 1981; XIA et al., 2007) and the $f-k$ transforms (e.g., LAcoss et al., 1969; Nolet and PANZA, 1976; YILMAZ, 1987), with their respective variants (e.g., CAPON 1969; LuO et al., 2008). The input signal can be either from an artificial controlled source, like in the Multi-Channel Analysis of Surface Waves (MASW) method (PARK et al., 1999), or the natural ambient vibration, like in Refraction microtremor (ReMi) (LouIE, 2001). All of these methods present some advantages and disadvantages, and thus it is difficult to generalize which could be optimal at a specific site and for a specific task. For example, $\tau-p$ methods perform generally better than $f-k$ methods for short duration 
transient signals, but have limited frequency resolution. Conversely, $f-k$ methods are more sensitive to uncorrelated noise. This is crucial, especially for the identification of surface wave higher modes (STROBbia, 2003). In this paper, we combine the features of the two approaches into a hybrid time-frequency-wavenumber method, based on the continuous wavelet transform (Goupillaud et al., 1984; Daubechies, 1990). The wavelet analysis has been already widely used in seismic surface wave analysis (e.g., КIM and PARK, 2002; KRITSKI et al., 2002; HolsChNEIDER et al., 2005; Kulesh et al., 2008) to study the nonstationary characteristics of the seismic wave field.

In the proposed technique, the seismic recordings are band-pass filtered using wavelet decomposition. The complex spectrogram is therefore computed for each trace separately. This gives the possibility to localize in time the instantaneous phase of all wave contributions, in the different analyzed frequency bands. This results also in an alternative way of performing active seismic acquisition, which allows the use of continuous recordings, for example, from seismological-like equipment (Figs. 1, 2).

Such an approach arises from the practical need to optimize the use of the available instruments for passive acquisition of natural vibrations, with that of

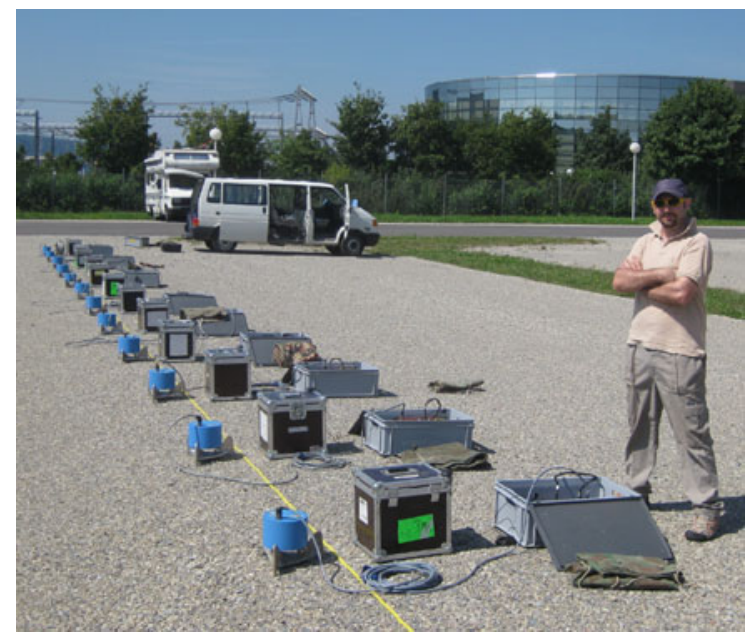

Figure 1

Example of a linear array of seismological stations during a hybrid seismic acquisition survey. Each station is an independent high resolution recording unit investigating shallower velocity structures. In surface wave analysis the resolution in depth is controlled by the frequency range of investigation and the seismic velocity structure of the site (AKI and RICHARDS, 1980). Ambient noise, in general, is suitable for the investigation at relatively low frequencies only, roughly $<10$ to $20 \mathrm{~Hz}$, (Horike, 1985; Louie, 2001). This is mostly because of the strong attenuation of the wave field generated by distant and low energy sources (natural or anthropogenic). To improve the resolution at shallower depths, high energy artificial sources have to be used (PARK et al., 2005). This gives the possibility of exciting surface wave higher modes, which are rarely identified with passive seismic waves (PoGGI and FäH, 2010). The proposed method has been tested on controlled source seismic records, both synthetics and real, for which we provide example results. For the real case, then, results from a combined active-passive survey performed in Lucerne (Switzerland) are also presented in the last section.

In the present study, additionally, we propose a new complex mother wavelet type, based on a raisedcosine envelope function (or Hanning taper), as an alternative to the classic Morlet wavelet. In the wavelet transform, the trade off between resolution in time and frequency is controlled by the type of mother wavelet employed. Compared to the simple Morlet type (FARGE, 1992), the proposed wavelet is advantageous because it allows an increased resolution in time, since it satisfies the admissibility conditions (DAUBECHIES, 1990) for smaller values of the nondimensional central frequency (see SHENG, 1995 for more details about wavelet properties).

\section{Method}

With standard $f-k$ power spectral methods like classical beamforming (LAcoss et al., 1969), the signal covariance matrix is obtained, at specific frequencies, from the complex conjugate cross-products between Fourier-transformed signals over the different offset distances. Thus, if a single wave propagates with a certain phase velocity, the elements of the covariance matrix will provide the relative phase delay information between all receiver pairs. If several waves propagate together but with slightly dissimilar phase velocities, 


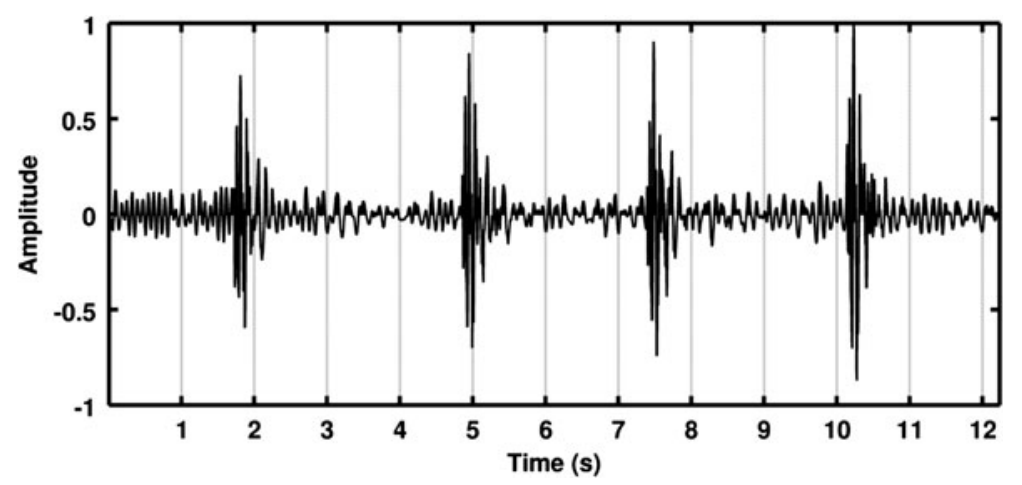

Figure 2

Example of multiple shots on a continuous recording of seismic data using seismological equipment. The shot receiver distance is $8 \mathrm{~m}$ in this example. The time synchronization between different stations is achieved by means of GPS time-stamping

then such an approach can hardly separate the different phase contributions. We propose, therefore, a different way to estimate the covariance matrix. We make use of time-frequency analysis using the continuous wavelet transform (GoupIlLaud et al., 1984; DaubeCHIES, 1990) to account for travel time delays induced by wave propagation over the different offsets. Basically, the covariance matrix is obtained by extracting and correlating only those values of the complex spectrogram that satisfy a specific velocity of propagation. The procedure is similar to a $\tau-p$ analysis, but applied here to the estimation of the instantaneous phases.

For a given frequency and velocity, then, the individual elements of this covariance matrix can be phase corrected to a common (and relative) reference time, according to the relative travel time delays. In case of multiple shots, moreover, successive covariance matrix estimates can be stacked and averaged, to enhance the phase delay estimation with respect to background uncorrelated noise. Finally, the $f-k$ energy spectrum can be computed, using either the beamforming technique (LAcoss et al., 1969) or any other high resolution method, based for example on data weighting (e.g., BURG, 1967; CAPON, 1969) or signal eigendecomposition (e.g. SснміDт, 1986). In the following sections, the different processing steps are presented separately to illustrate the basics of the proposed method.

\subsection{Multiple Shot Triggering of Continuous Records}

Continuous recording comes from the need to optimize the use of our acquisition equipment for passive seismic in combination with active experiments. The main practical disadvantage in using seismological stations lies in the difficulty of triggering at the initial time $\left(t_{0}\right)$ of the artificial source. In practice, in all approaches based on cross-correlations (in the time or the frequency domain) the knowledge of an absolute $t_{0}$ is not necessary, since only the relative phase delay information between traces is analyzed. For each shot, then, a simple relative reference initial time can be used instead of an absolute one.

To estimate the relative reference time $\left(t_{0}, t_{1}, \ldots\right.$, $t_{n}$ ) of the $n$ consecutive shots of a given reference trace $u\left(x_{\text {Ref }}, t\right)$ (generally the first offset distance), we use an automatic triggering procedure based on the analysis of the envelope function of the analytical representation of the signal (Fig. 3). In this way, the first $n$ most energetic amplitudes of the trace can be collected and sorted according to their energy levels. By contrast to simpler approaches such as the Short Term Averaging/Long Term Averaging (STA/LTA) algorithm, the advantage in using this procedure is the minimization of bias introduced by the random phase interaction, which might produce peaks at different relative times of the consecutive shots.

\subsection{Travel-Time Covariance Matrix Estimation}

In all beamforming type techniques (classical or high resolution), the signal covariance (or cross-spectral) matrix has to be computed over the different discrete frequencies to estimate the $f-k$ energy spectrum. Thus, a Fourier analysis of the recordings is required. 


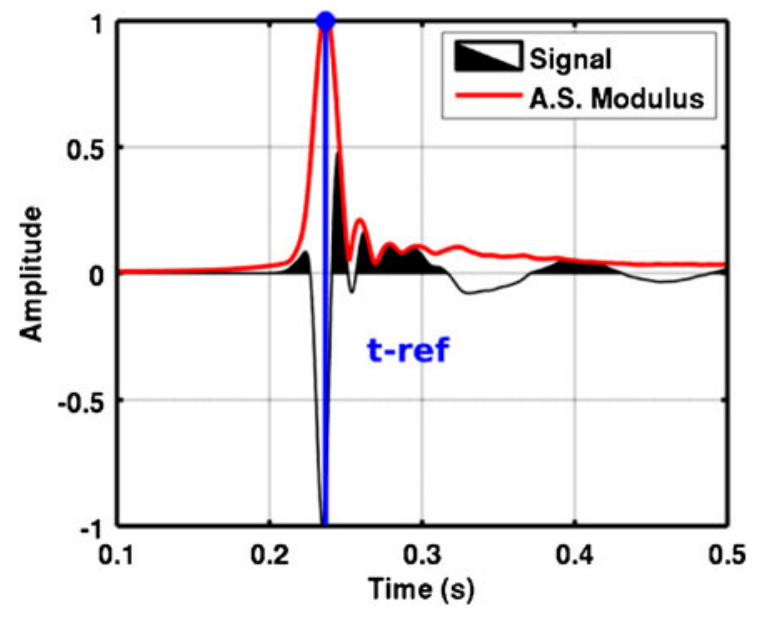

Figure 3

Automatic triggering of a single shot reference time ( $t$-ref, in blue) using the modulus amplitude of the complex analytical signal (A.S., in red). The obtained $t_{0}$ is the relative reference for phase correction, but it does not represent the true shot time

If we indicate with $s$ the complex Fourier spectrum of the recorded signal $u$ at the frequency $f$ and offset $x$ as:

$$
s(f, x)=\int_{-\infty}^{\infty} u(x, \tau) e^{-2 \pi j f \tau} \mathrm{d} \tau
$$

and the column vector of spectra at different offsets as:

$$
S(f)=\left[s\left(f, x_{1}\right), \ldots, s\left(f, x_{n}\right)\right] .
$$

Then the covariance matrix can be simply estimated as:

$$
\hat{R}(f)=E\left\{S(f) \cdot S(f)^{h}\right\}
$$

where $h$ stands for Hermitian complex conjugate.

Usually, the whole record window is used for the computation of the Fourier spectra. In such a way, however, the influence of body waves and other contributions (e.g., noise, air blast) can significantly affect the final result, introducing some bias in the phase estimate of surface waves. To avoid this problem, it is common procedure to manually select the appropriate windows (tapering in the time offset domain) to exclude the direct and refracted arrivals and to emphasize the surface wave content. Such an approach, however, is influenced by the subjectivity of the operator who defines the window, since no strict rules are (and can be) established for this procedure. In some cases, portions of the traces cut out by manual windowing can still contain usable surface wave information. Moreover, the length of the selected windows can be very different at different offsets, affecting the robustness of the estimates of the covariance matrix elements.

\section{Time (s)}

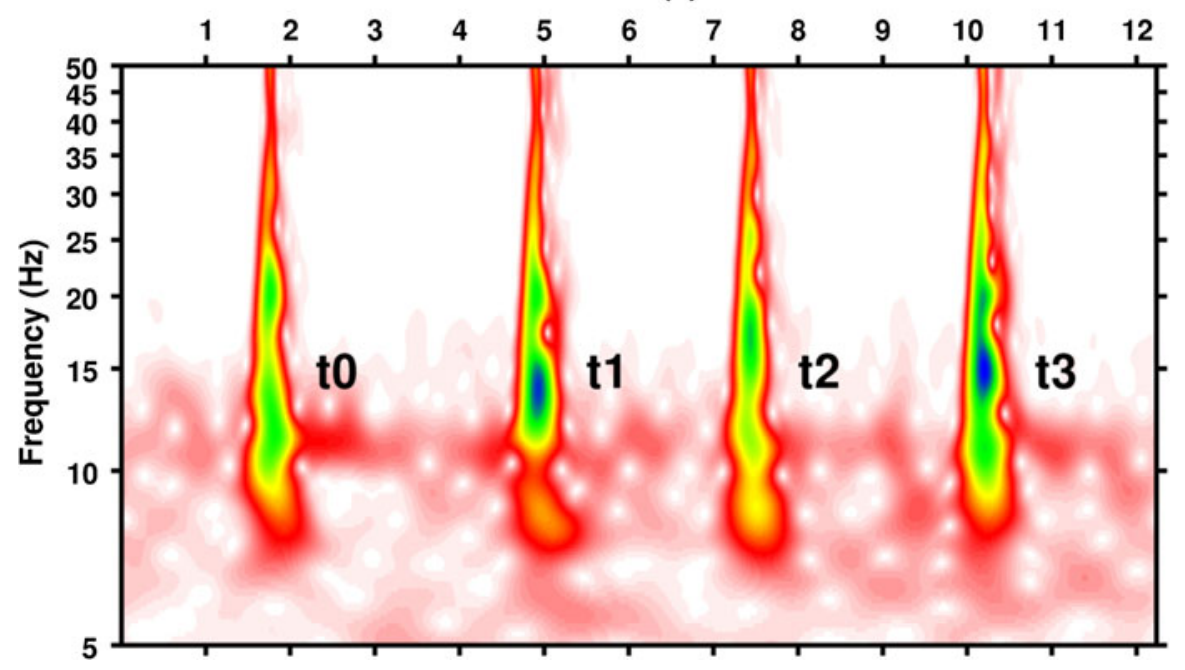

Figure 4

Wavelet spectrogram (magnitude) of four shots recorded at the first receiver location. The raised-cosine mother wavelet was used for this example 


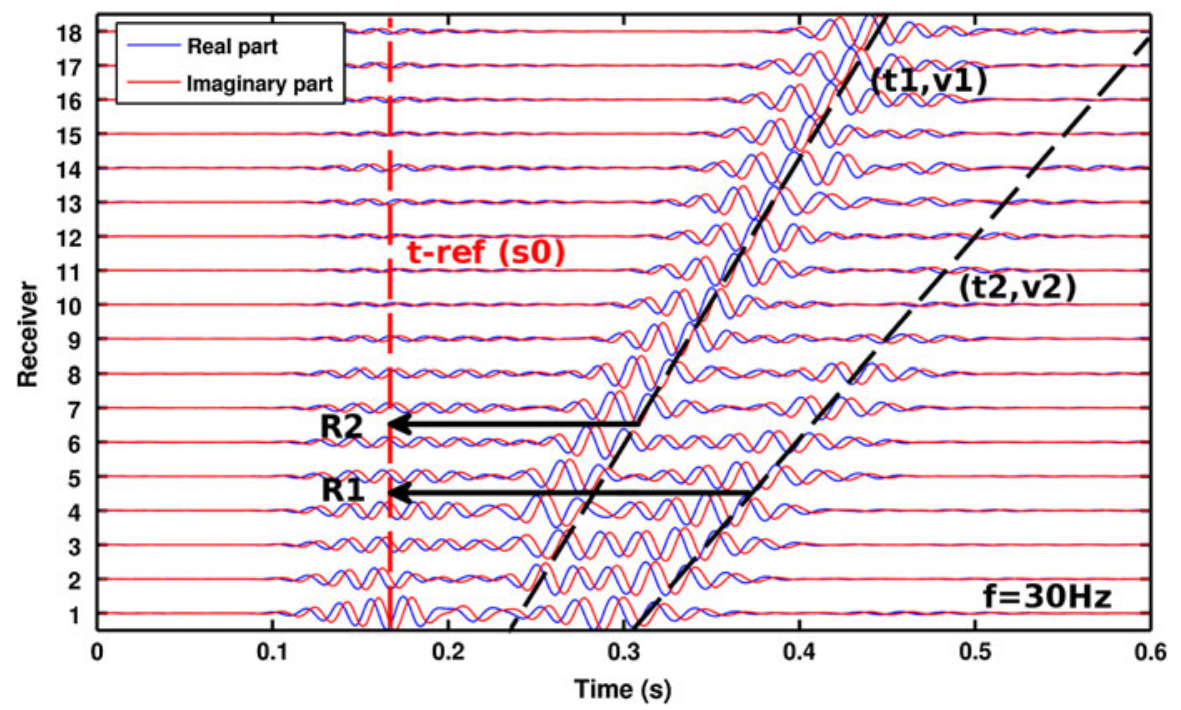

Figure 5

Wavelet transformed traces (real and imaginary part) of a 18 stations seismic record (here filtered at $30 \mathrm{~Hz}$ ). It is possible to follow two separate wave packets propagating with different source delay time and phase velocity $(t, v)$. The triggered reference time ( $t$-ref) for a given shot $\left(s_{0}\right)$ is used for travel-time correction of the covariance matrix elements $(R)$ for the investigated phase velocity

To overcome this problem, we perform a timefrequency analysis of the records, using the continuous wavelet transform (Fig. 4). This approach has the advantage to make the phase information separable in time (as instantaneous estimation) and thus for the different wave contributions. Therefore, once a propagating wave is identified, its instantaneous phase can be extracted at a specific frequency. Clearly, the quality of the result is controlled by the trade off between the resolution in time and frequency of the wavelet transform. In general, the higher the resolution in time, the lower the frequency, and vice versa. The time-bandwidth product is nevertheless constant.

The problem is how to automatically isolate a particular wave on the complex spectrogram obtained from the wavelet decomposition. For surface waves this cannot be simply done by travel-time picking, since the surface-wave arrival-time cannot be localized because of the dispersion. To solve this, we implemented a direct search approach, based on the idea of $\tau-p$ analysis (or the slant-stack), but applied here to wavelet filtered signals. If we define the wavelet transform $w$ of the signal $u$ at a specific frequency $f$ and offset $x$ as:

$$
w(f, x, t)=\int_{-\infty}^{\infty} u(x, \tau) w_{m}^{h}(f, t, \tau) \mathrm{d} \tau
$$

where $w_{m}$, is the filter bank base to be used (or the mother wavelet, see Sect. 3), then the offset-vector obtained by those complex values that satisfy a specific velocity of propagation $v$ and source delay time at the different offsets can now be written as:

$$
\begin{aligned}
S(f, t, v)= & {\left[w\left(f, x_{1}, t+\frac{\left(x_{1}-x_{\mathrm{Ref}}\right)}{v}\right), \ldots,\right.} \\
& \left.w\left(f, x_{n}, t+\frac{\left(x_{n}-x_{\mathrm{Ref}}\right)}{v}\right)\right]^{\mathrm{T}}
\end{aligned}
$$

Therefore, the covariance matrix is:

$$
\hat{R}(f, t, v)=E\left\{S(f, t, v) \cdot S(f, t, v)^{h}\right\} .
$$

In practice, $\hat{R}(f, t, v)$ is computed as the Hermitian cross-products between those instants on the complex spectrograms that correspond to a specific wave (see examples in Fig. 5). With respect to the classic way of estimating the covariance matrix-using Fourier transform-the correlation depends now on three independent variables and thus is travel-time dependent. However, since the $t$ and $v$ parameter pairs are not 
known a priori, the matrix has to be recomputed for every possible combination, within a given reasonable range of expectation. The delay time search parameter $t$, in particular, is necessary because we might expect the surface waves not to develop immediately at the shot time, and higher modes not to be simultaneous with the fundamental.

As a last step, each element of $\hat{R}(f, t, v)$ should be phase-corrected back to a reference time common to all traces (e.g., $t$-ref obtained by triggering), to compensate for the effect of travel-time delay over the different offsets:

$$
\hat{R}_{i, j}^{\phi}\left(f, t_{\mathrm{Ref}}, v\right)=\hat{R}_{i, j}(f, t, v) \cdot e^{\left[-2 \pi f\left(t_{\mathrm{Ref}}+\frac{\left(x_{j}-x_{i}\right)}{v}\right)\right]} .
$$

With this completed, it is now possible to use such covariance matrix estimate to compute the $f-k$ energy spectrum with standard beamforming algorithms.

\subsection{Covariance Matrix Stacking and Phase- Averaging}

The classic acquisition protocol for active seismic surveys includes the stack of consecutive recordings to improve the signal-to-noise ratio. This is particularly suitable for reflection/refraction seismic, where only the correct identification of the travel-times is of primary importance, but it might not be strictly necessary for surface wave analysis and in case of continuous recordings. Averaging the phase over different shots implies the assumption that each wave excitation will produce exactly the same phase spectrum at the source. This assumption, however, might not be perfectly fulfilled in reality when simple artificial sources are used, like the mini-gun or the sledge hammer. For these devices it is indeed difficult to ensure that they will always operate in a repeatable fashion. This is particularly evident at rather high frequency ranges, where we can observe phase cancellation.

To enhance the final resolution of the $f-k$ estimate, instead of averaging the single phase estimates, we average the phase differences between receiver pairs. This can be done by stacking the travel-time corrected covariance matrix over $N$ consecutive shots:

$$
\left[\hat{\mathbf{R}}^{\phi}(f, t, v)\right]_{\mathrm{TOT}}=\sum_{m=1}^{N(\text { shots })} \frac{\left[\hat{\mathbf{R}}^{\phi}(f, t, v)\right]_{m}}{N} .
$$

Such a procedure minimizes the effect of uncorrelated noise, enhances the phase delay estimation and stabilizes the covariance matrix for the use with high resolution $f-k$ algorithms based on eigendecomposition (e.g., multiple signal classification or MUSIC).

\subsection{Note on Amplitude Normalization}

Due to geometrical and intrinsic attenuation, the wave amplitude decreases with increasing distance from the source. If the maximum interdistance between receivers is too large, an offset normalization procedure might be necessary to emphasize the information at far offsets. We tested different approaches to equalize the signal energy content at the different receiver locations.

As a first attempt, we applied a simple geometrical decay correction approach, assuming that to a first approximation surface waves attenuate with distance $r$ as $1 / \sqrt{r}$. Such as approach, however, doesn't take into account the influence of anelasticity and explains only geometrical spreading in the farfield for vertically heterogeneous media. A second possibility consists in normalizing the individual traces with respect to their relative energy levels. This can be done by dividing by the maximum amplitude or, more accurately, by normalizing with respect to the whole energy content of a trace:

$$
w(f, x, t)=\frac{w(f, x, t)}{\left[\int|w(f, x, t)|^{2} \mathrm{~d} t\right]^{1 / 2}} .
$$

In such a case the correction is independent of any prior assumption but, when the energy content of body waves is too large (e.g., at near offsets), it might lead conversely to an underestimation of the surface wave contribution. Thus it is preferable to be used at intermediate to large offsets only.

Such procedures have been tested both on raw and wavelet filtered traces, for comparison. As expected, correcting for geometrical spreading provides comparable results in the two cases, as only a constant multiplicative factor is applied to a linear 
transformation. However, differences are relevant in the case of amplitude/energy equalization, which provides better results only when used on wavelet filtered traces. This is most probably related to the removal of uncorrelated noise by the filtering, which is thus not subsequently amplified by the correction as it would be on raw traces.

Finally, an interesting alternative consists in directly normalizing the signal covariance matrix using the approach proposed by CAPON (1969). In this case, the normalization is based on the relative amplitude of each pair of cross-correlated signals:

$$
\hat{R}_{i, j}^{\phi}(f, t, v)=\frac{\hat{R}_{i, j}^{\phi}(f, t, v)}{\left[\hat{R}_{i, i}^{\phi}(f, t, v) \hat{R}_{j, j}^{\phi}(f, t, v)\right]^{1 / 2}}
$$

for $i \neq j$, and:

$$
\hat{R}_{i, j}^{\phi}(f, t, v)=1
$$

for $i=j$.

After testing, we found the latter approach convenient in combination with energy normalization to enhance the detection of higher modes at intermediate to large offset distances. This procedure is therefore used in all following tests.

\subsection{The f-v-t Power Spectrum and Grid Search}

Applying the above described procedures, we obtained estimation of the signal covariance matrix that depends, rather than on frequency, but on the analyzed phase velocity and source time of the surface waves. The $f-v-t$ power spectrum can be directly computed using the classic tools of $f-k$ analysis, e.g., like classic beamforming:

$$
P(f, t, v)=\frac{e(f, v)^{h} \hat{R}^{\phi}(f, t, v) e(f, v)}{N^{2}}
$$

high-resolution beamforming:

$$
P(f, t, v)=\frac{1}{e(f, v)^{h}\left[\hat{R}^{\phi}(f, t, v)\right]^{-1} e(f, v)}
$$

or the MUSIC algorithm:

$$
P(f, t, v)=\frac{1}{e(f, v)^{h}\left[\hat{U}(f, t, v) \hat{U}(f, t, v)^{h}\right] e(f, v)}
$$

where $e(f, v)$ is the steering vector of the $v-f$ search, and $\hat{U}(f, t, v)$ consists of the subset of eigenvectors of the covariance matrix $\hat{R}^{\phi}(f, t, v)$ that span the noise subspace.

However, the results from all high resolution methods strongly depend on specific site-related conditions, such as the amount of uncorrelated noise and the local accuracy in the phase estimation. These methods, moreover, do not always provide accurate results in cases of multiple overlapping signals, as, for example, with higher mode identification. Therefore, to better stress the possibilities and limitations of our methodology, in the following sections we will present the results using the classical beamforming algorithm only.

From the implementation point of view, identifying and extracting the surface dispersion curves is done by means of a power spectrum local maxima search over a three-dimensional parameter space. For simplicity, we first perform a grid search over $t$, with fixed $v$ and $f$ (Fig. 6). The procedure is then repeated for all combinations of $v$ and $f$, respectively. Obviously, such processing is computationally more expensive than the classic approaches for surface wave analysis. However, the entire procedure can be conducted automatically and, even for a high number of shots, without the intervention of the user.

\section{The Raised-Cosine Mother Wavelet}

To compute the time-frequency spectrogram, the continuous wavelet transform is used. Since we are dealing with phase delay estimation, a complex mother wavelet has to be adopted. Amongst the possible choices, a very common mother wavelet in seismology is the Morlet (GoupILlaud et al., 1984). This can easily be obtained from the convolution between a harmonic complex signal of given frequency, with a Gaussian envelope, whose width controls the tradeoff between time and frequency resolution of the wavelet. Its nondimensional time $\eta$ representation is:

$$
w_{m}(\eta)=\pi^{-1 / 4} e^{-\eta^{2} / 2} e^{j C_{0} \eta},
$$

and in normal time $t$ : 


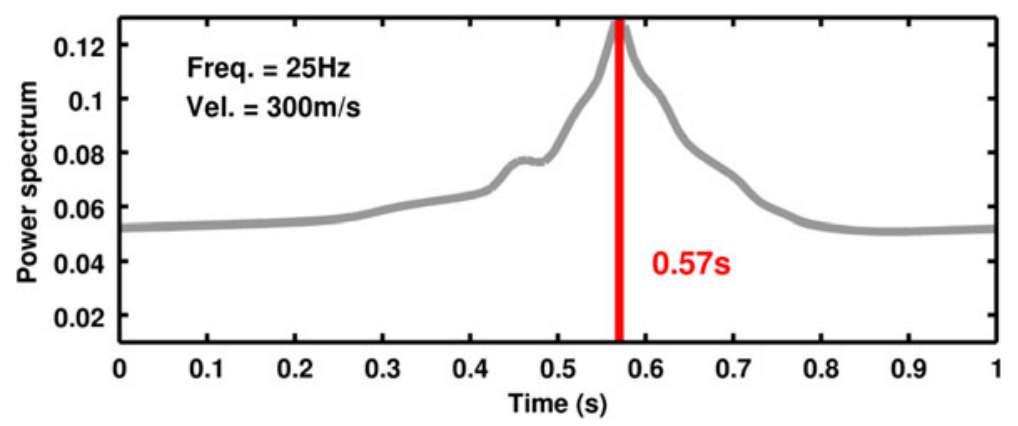

Figure 6

Power spectrum search over surface wave source time, fixed velocity and frequency. In this example, the maximum of correlation between signals is reached about $0.57 \mathrm{~s}$ after the triggered $t$-ref of the shots. This corresponds to the second higher mode of Rayleigh waves

$$
w_{m}(t)=\pi^{-\frac{1}{4}} e^{-\frac{1}{2}\left(\frac{\omega_{0} t}{C_{0}}\right)^{2}} e^{j \omega_{0} t} .
$$

It is well known, however, that the Morlet wavelet does not always satisfy the admissibility conditions (FARGE, 1992) for any value of the wavelet coefficient $C_{0}$ (or the non-dimensional central frequency). For values of $C_{0}$ lower than 6 , the wavelet spectrum starts to diverge. Consequently, such a wavelet presents a lower resolution bound in time, which makes difficult the analysis of extremely narrow impulsive transients. This is generally not an issue in most seismological applications, but can be a limitation when separation of short transients close in time is required.

To partially overcome this problem, we propose an alternative-although similar type-of complex wavelet, based on a simple raised-cosine tapering window (or Hanning type) applied to a complex exponential function (Fig. 7). The wavelet can be expressed in the nondimensional time $\eta$ as:

$$
w_{r c}(\eta)=\frac{1}{2}(1+\cos (\eta)) e^{j C_{0} \eta} \quad \text { for }-\pi \leq \eta \leq \pi,
$$

and in normal time $t$ as:

$$
\begin{gathered}
w_{r c}(t)=\frac{1}{2}\left(1+\cos \left(\frac{\omega_{0} t}{C_{0}}\right)\right) e^{j \omega_{0} t} \\
\text { for }-\frac{\pi C_{0}}{\omega_{0}} \leq t \leq \frac{\pi C_{0}}{\omega_{0}}
\end{gathered}
$$

where $\omega_{0}$ is the angular central frequency.

The proposed wavelet presents some differences compared to the Morlet wavelet. Its energy is entirely
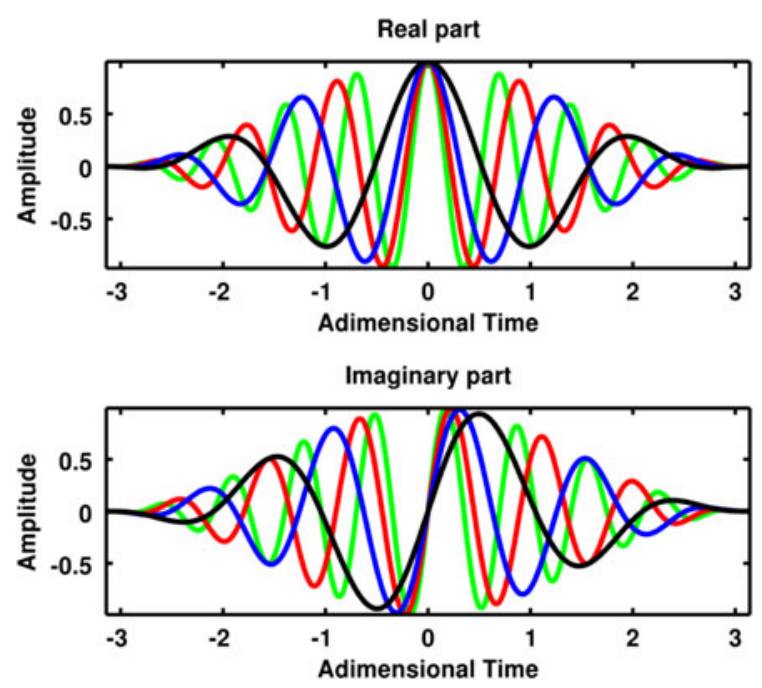

Figure 7

Real (on top and imaginary (on bottom) part of the raised-cosine mother wavelet, computed for nondimensional central frequency $C_{0}$ values of 1, 2, 3, 4 (black, blue, red and green, respectively)

bounded in time $(-\pi \leq \eta \leq \pi)$ and has an admissibility condition satisfied for any value of $C_{0}$ being integer and higher than 1 :

$$
\int_{-\pi}^{\pi} w_{r c}(\eta) \mathrm{d} \eta=0 \quad \text { for } C_{0} \in(N \geq 2) .
$$

Therefore, it allows an increased time resolution, which is indeed not possible with the standard Morlet wavelet. For values of $C_{0}$ equal and higher than 6 , nevertheless, the results from the two wavelets become progressively comparable (e.g., Fig. 8). The Fourier spectrum of such wavelet can be easily obtained in the following analytical form as: 


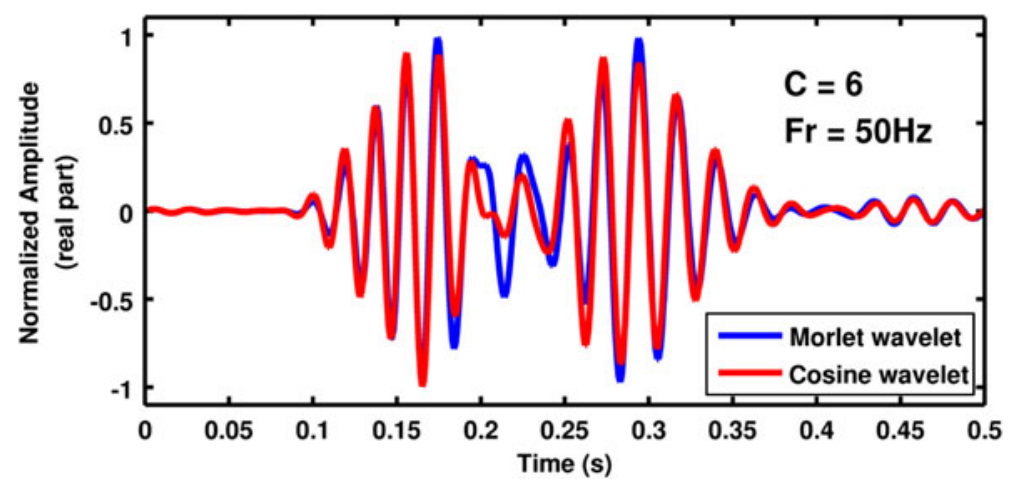

Figure 8

Comparison of wavelet-filtered trace (real part) using the classic Morlet (in blue) and the raised-cosine wavelets (in red). A relatively low value of the nondimensional central frequency $C_{0}$ is used

$$
\begin{aligned}
w_{r c}(\omega)= & -\left[\frac{\omega_{0}^{2}}{\left(\omega_{0}-\omega\right)^{3} C_{0}^{2}-\omega_{0}^{2}\left(\omega_{0}-\omega\right)}\right] \\
& \times \sin \left(\frac{\left(\omega_{0}-\omega\right) C_{0} \pi}{\omega_{0}}\right) .
\end{aligned}
$$

Compared to the Morlet wavelet, the spectrum is in this case sharper, for the same values of the central frequency $C_{0}$ (Fig. 9), even though it presents typical low energy side lobes and zeros.

\section{Results}

To test the robustness and utility of the proposed algorithm, both synthetic and real datasets were analyzed. In all cases, three component recordings were used, in order to analyze the different surface wave contributions (both Love and Rayleigh waves).

\subsection{The Synthetic Dataset}

A set of synthetic seismograms were generated for a previous active seismic study (SCHULER, 2008). For the modeling, an algorithm originally written by Friederich and Dalkolmo (1995) was used. The model, taken from the literature (DAL Moro et al., 2006), consists of three horizontal layers with seismic velocities $\left(V_{\mathrm{s}}\right.$ and $\left.V_{\mathrm{p}}\right)$ progressively increasing with depth (Table 1). A vertical point (delta) source located at the surface was employed, with frequency bandwidth of 1-60 Hz. Each synthetic consists of the continuous recording of 4 consecutive shots, each of
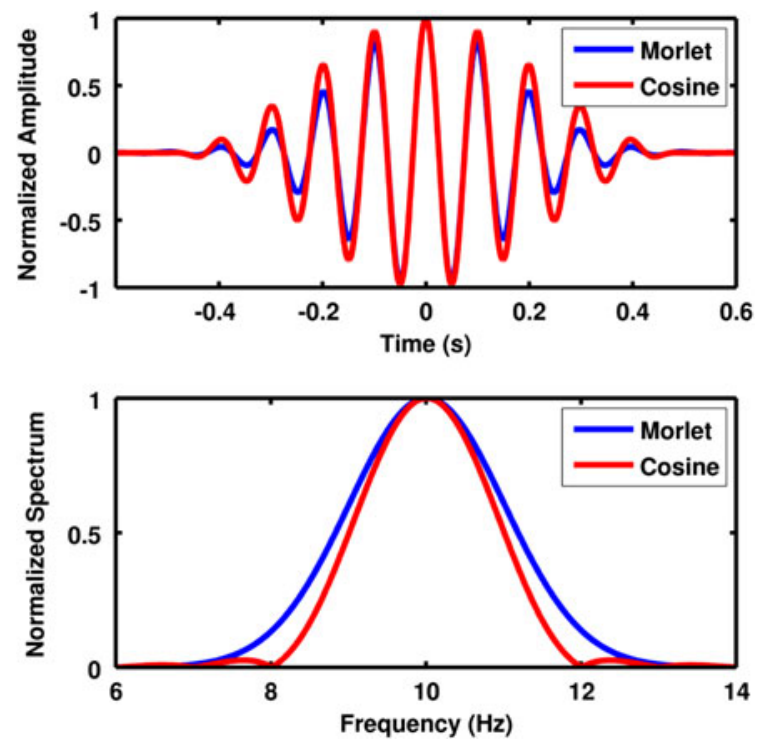

Figure 9

Comparison in time (on top, real part) and frequency (on bottom, absolute value) domains between the Morlet and the raised-cosine wavelets. In the example, values of $C_{0}=10$ and $\omega_{0}=10 \mathrm{~Hz}$ were used

$2 \mathrm{~s}$ duration. To emulate realistic field conditions, a considerable amount of white (uncorrelated) noise was applied to the traces before processing. Synthetics seismograms were generated for 40 receiver locations at $2.5 \mathrm{~m}$ spacing.

For comparison, we processed the recordings using both the classic Fourier-based method and the wavelet approach (Figs. 10, 11) using beamforming. In particular, the two methods always provide comparable results in the case of a single shot and 
Table 1

Parameters of the one-dimensional models employed to generate active seismic synthetics

\begin{tabular}{llccc}
\hline & $\begin{array}{l}\text { Thickness } \\
(\mathrm{m})\end{array}$ & $\begin{array}{l}\text { P-velocity } \\
(\mathrm{m} / \mathrm{s})\end{array}$ & $\begin{array}{l}\text { S-velocity } \\
(\mathrm{m} / \mathrm{s})\end{array}$ & $\begin{array}{r}\text { Density } \\
\left(\mathrm{kg} / \mathrm{m}^{3}\right)\end{array}$ \\
\hline Layer 1 & 8 & 340 & 140 & 1,700 \\
Layer 2 & 8 & 2,770 & 1,570 & 2,050 \\
Layer 3 & - & 5,200 & 3,000 & 2,400 \\
\hline
\end{tabular}

in absence of noise disturbances. However, when consecutive shots are used and Gaussian noise applied, the wavelet method produces more stable results (Figs. 10b, 11b) in comparison to the classic approach (Figs. 10a, 11a) which considerably deteriorates. Specifically, the higher modes are better emphasized, even at high velocities, and therefore mode readily identifiable (Fig. 12). This can be explained by the more robust estimation of phase delays obtained with the $t-v-f$ grid search combined with the previously discussed procedures of covariance matrix stacking and normalization, in the presence of strong uncorrelated noise.

\subsection{Testing Real Data: Lucerne (Switzerland)}

To test the method on a real case, a joint activepassive seismic survey was performed in Lucerne (Switzerland). The target of our analysis is a wellstudied area, which has been extensively investigated with passive seismic techniques as part of a previous microzonation study (PogGi et al., 2012). However, the sole use of passive techniques did not provide sufficient resolution at shallow depths, which are significant to characterize the high frequency seismic response during an earthquake. Together with the analysis of boreholes logs, an uppermost low seismic velocity layer was identified. This layer appears to be continuous over most of the survey area, but with a variable thickness. Due to the strong velocity contrast with the deeper structure, this layer suits the conditions for generating a sufficient amount of surface waves during an active seismic experiment.

A test was performed combining a passive and an active seismic acquisition survey. For the experiment, 12 seismological stations were used, equipped with triaxial velocity sensors (5 $\mathrm{s}$ natural frequency). For the active seismic test, a linear configuration was implemented (Fig. 13), with $4 \mathrm{~m}$ spacing between receivers. According to the available number of stations and the type of sensors, this configuration limited considerably the resolution of the dispersion curves at high frequencies (roughly $40 \mathrm{~Hz}$ for the expected phase velocity range), but gave the possibility to extend the analysis to frequencies close to that of the passive acquisition. We tested different shot-offset distances $(2,5,10,20 \mathrm{~m})$. Due to the length of the deployment, however, the shorter offset provided the better results. Within each shot location, five consecutive wave-field excitations were performed, with about 1-2 s delay between them. As the source, we used a sledge hammer impacting on a special triangular base plate that gives the possibility of reproducing a $45^{\circ}$ point source. When aligned to the array deployment, such a device allows the generation of Rayleigh waves, including the radial component. This configuration is analyzed in this example.

The processing of the vertical and radial component data provided comparable results. For both cases, the power spectrum was computed in a frequency range between 5 and $40 \mathrm{~Hz}$ using the wavelet approach to estimate the covariance matrix, together with classical beamforming (Figs. 14, 15). This frequency range is sufficient for the identification of five Rayleigh wave modes that can be correctly assigned at least up the fourth overtone. Surprisingly, the fundamental mode that is generally the most energetic over a broad frequency range lacks energy above $10 \mathrm{~Hz}$ and cannot be reliably followed anymore. Conversely, the energy progressively distributes to the higher modes, which can easily be tracked up to $40 \mathrm{~Hz}$.

For the passive seismic acquisition, the stations were subsequently reorganized in a crossed configuration (see Fig. 13) along two main directions. Such a configuration is common in urban environments, because it adapts to the available space along crossroads. The total diameter of the array deployment was about $140 \mathrm{~m}$, which results, with the high resolution $f-k$ method, in a low resolution cut-off of about $3 \mathrm{~Hz}$ (see PoGGI and FÄH, 2010 for more details about the resolution in passive $f-k$ processing).

The result of the active seismic experiment has been compared with that from passive acquisition 

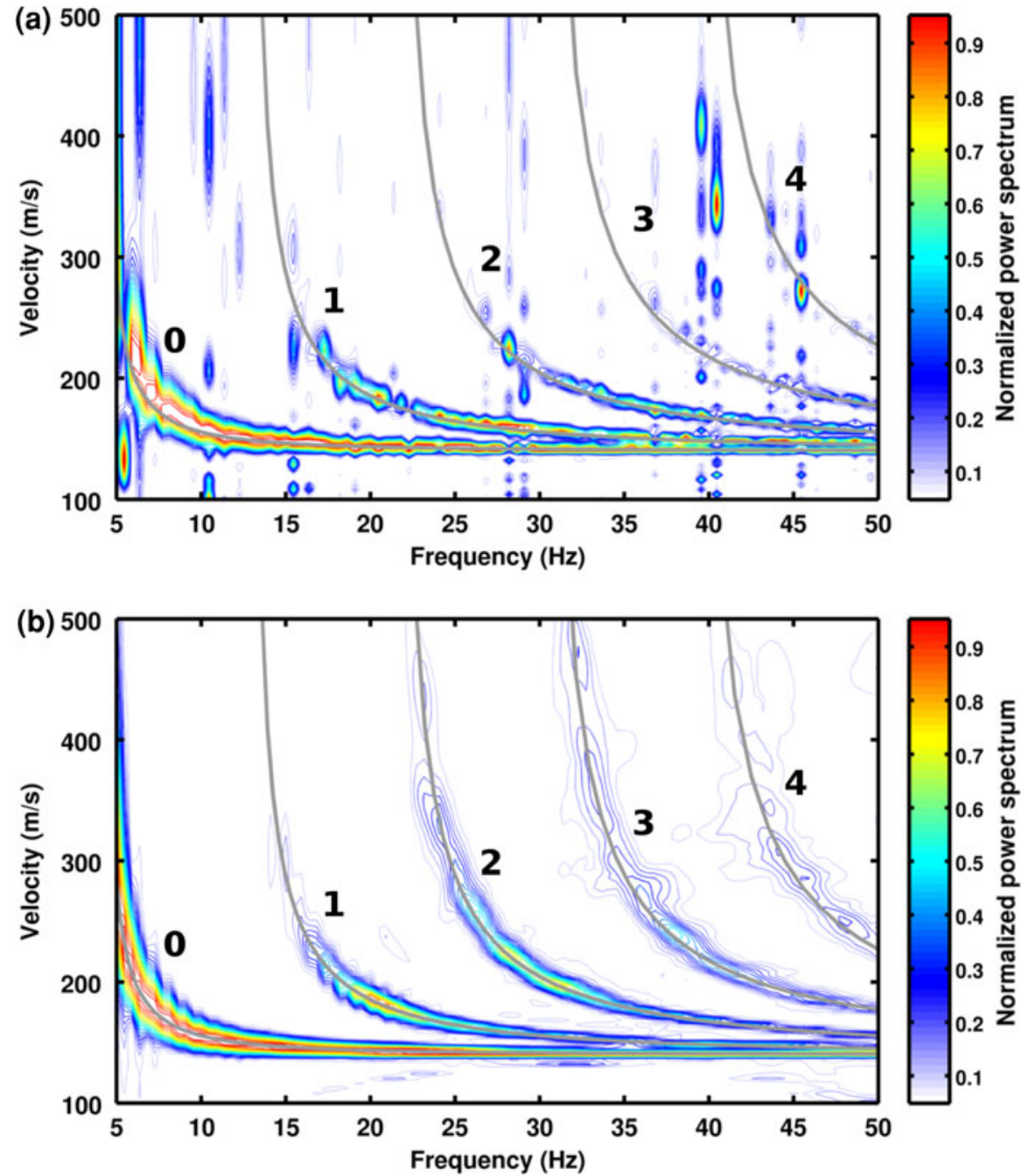

Figure 10

Frequency-velocity power spectrum of the synthetic dataset using the classic Fourier-based beamforming (a) and the wavelet method (b). In this example, the transverse ( $\mathrm{SH}$ ) component is shown, using 40 receivers at $2.5 \mathrm{~m}$ intervals. Four shots were performed. Here, other than the fundamental mode of Love waves, four additional higher modes can be clearly identifiable (the analytical solution is shown in the gray solid lines)

(Fig. 16). The processing of ambient vibrations was made using the three-component high resolution beamforming algorithm, as explained in PoGGI and FÄH (2010). Unfortunately, the processing of the two horizontal components (radial and transverse) did not provide usable results, probably caused by the difficult measurement environment. On the vertical component, however, it was possible to clearly identify the fundamental mode Rayleigh wave in a frequency range roughly between 3.5 and $6.5 \mathrm{~Hz}$. The curve is consistent with that portion of the fundamental mode identified by active seismic testing. The phase velocity jump at $7 \mathrm{~Hz}$ can be associated with the aforementioned presence of the low-velocity layer.

\section{Discussion and Conclusions}

In this paper we have presented a modified approach to surface wave analysis of active seismic experiments, based on the continuous recordings 

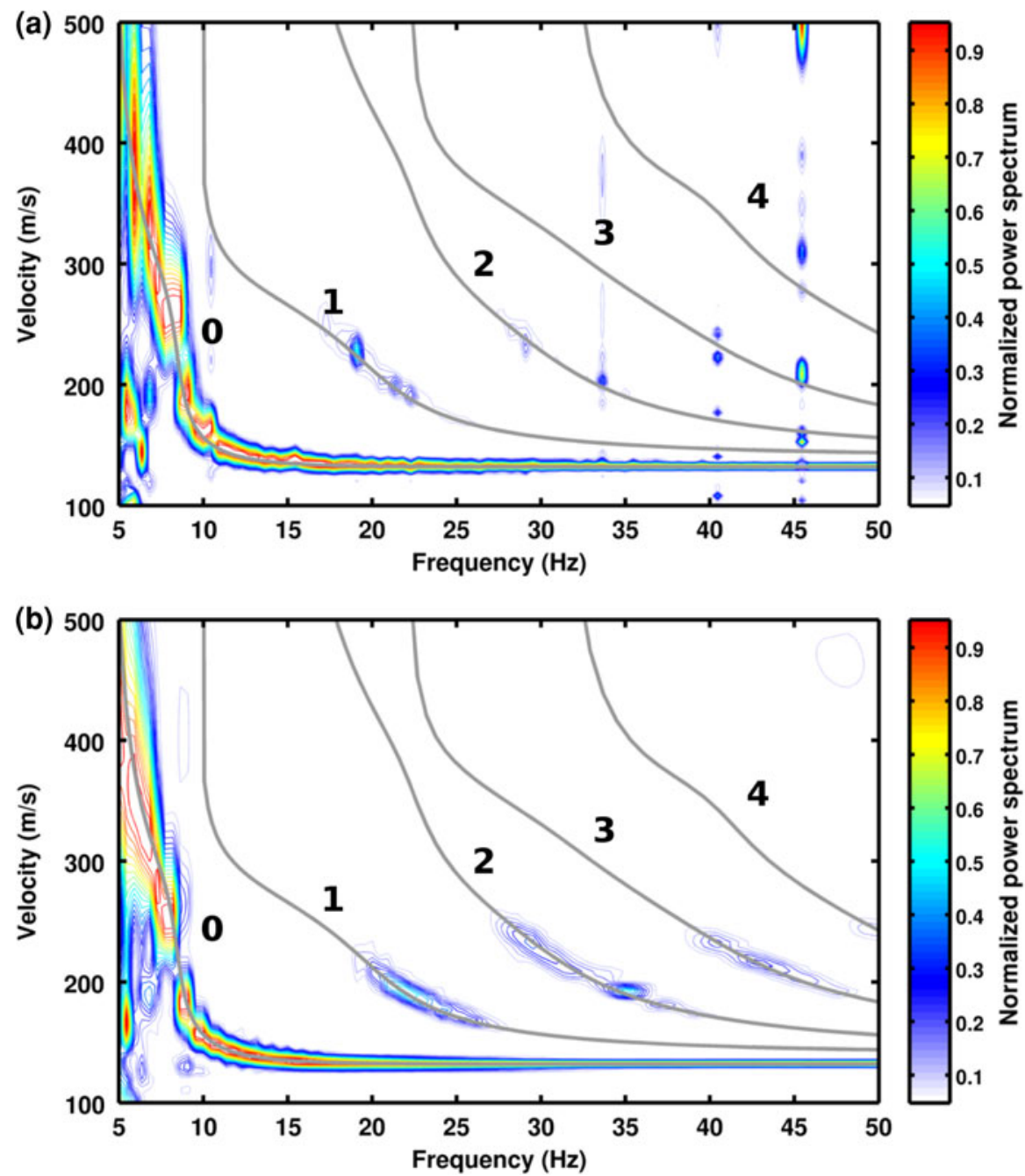

Figure 11

Frequency-velocity power spectrum of the synthetic dataset using the classic Fourier-based beamforming (a) and the wavelet method (b). In this example, the vertical (PSV) component is shown, using 40 receivers at $2.5 \mathrm{~m}$ intervals. Four shots were performed. In comparison to the transversal component in Fig. 10, the higher modes are here less energetic with respect to the fundamental, and consequently more difficult to track

from seismological stations. We use the continuous wavelet transform to extend the capability of beamforming techniques in detecting short transients that propagate in space with specific phase velocities. In practice, the proposed approach relies on the estimation of surface wave travel-times to enhance the estimation of the signal covariance matrix. Moreover, stacking the covariance matrix over consecutive wave field excitations enhances the imaging of surface wave dispersion in noisy environments. The method is therefore particularly advantageous for higher mode detection, which is generally more affected by uncorrelated noise disturbances.

We tested the technique on synthetic records, as well as on a real case in the city of Lucerne. In both cases, multiple modes of the surface waves were detected. In comparison to the classic Fourier-based beamforming, we obtained a better imaging of the dispersion pattern, through reduction of the effects of the local noise level. For the Lucerne experiment, moreover, the results are consistent with an estimation of the Rayleigh wave fundamental mode 


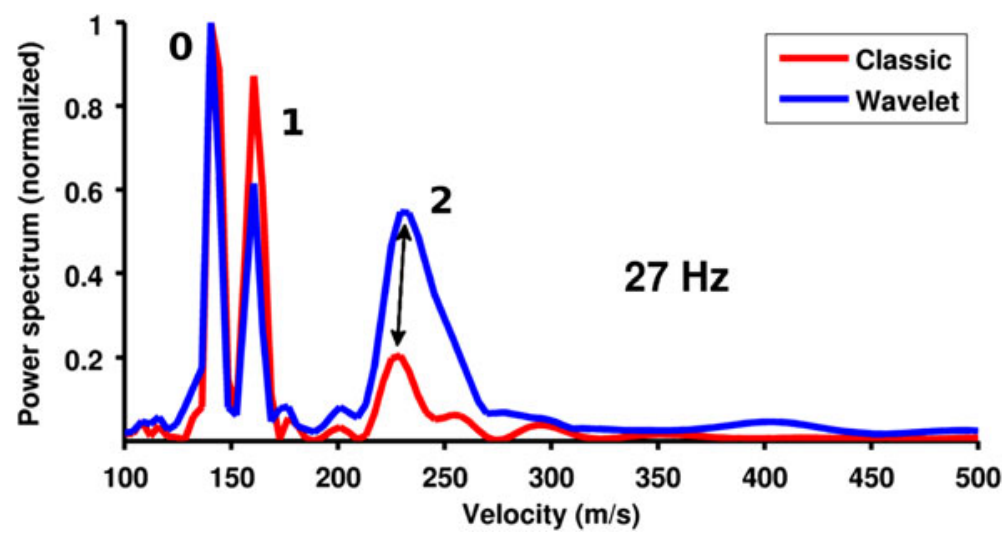

Figure 12

Comparison of the power spectrum computed with different approaches. Notice how the proposed wavelet-based method emphasizes the energy content of the higher modes (here for the transverse component), making them more visible (e.g., the second higher mode at about $230 \mathrm{~m} / \mathrm{s}$ )

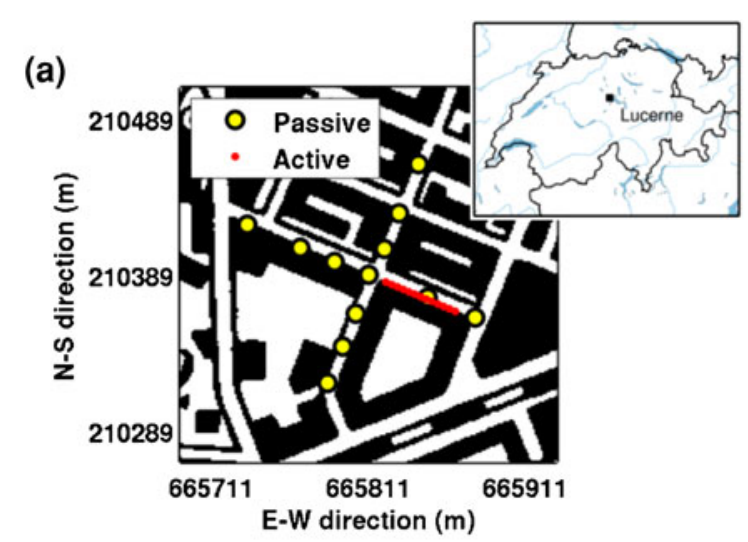

(b)

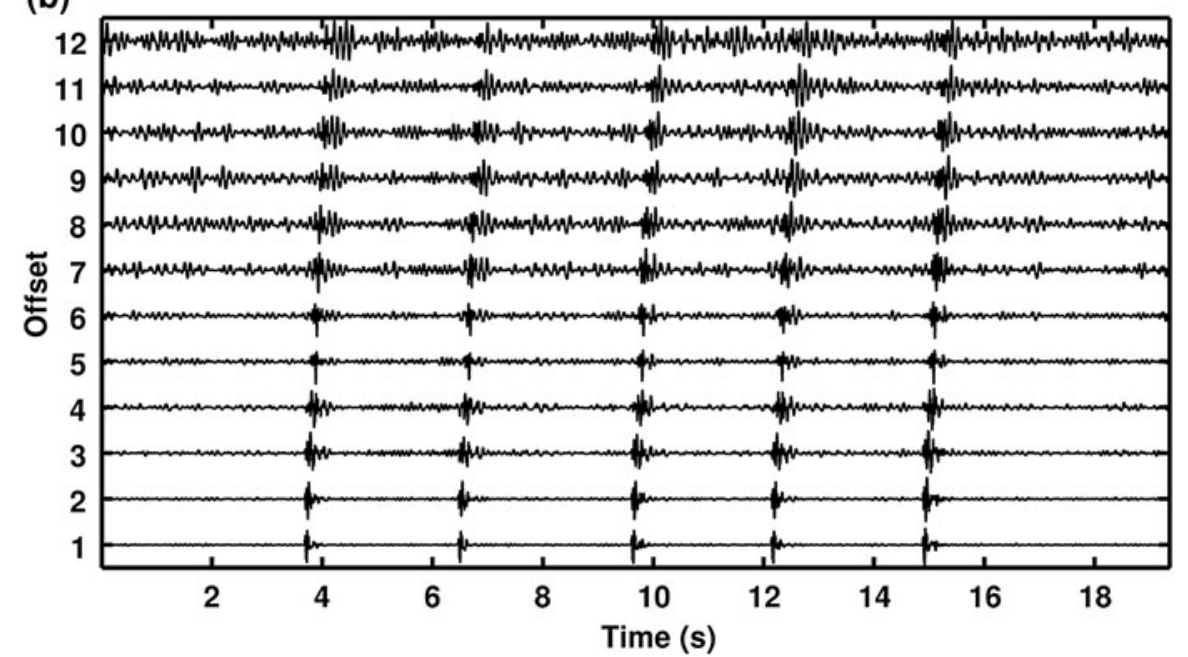

Figure 13

a Array geometry for the passive (crossed configuration) and active (linear) seismic acquisition. Units are in Swiss coordinates (CH1903). b Example of continuous recordings from the seismological stations during the active experiments. Several series of five consecutive shots were performed, with about 1-2 s delay between, using a sledge hammer 

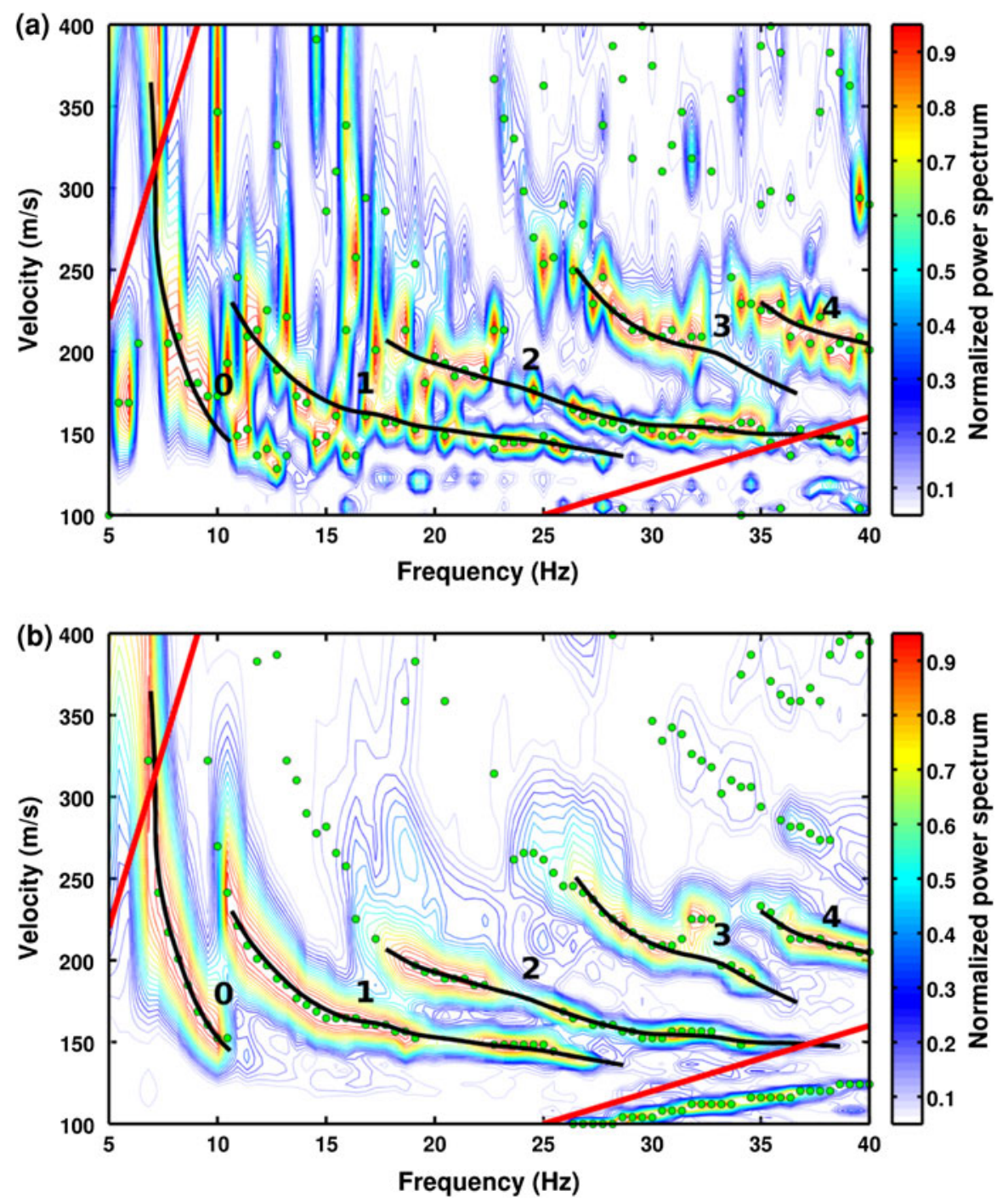

Figure 14

Frequency-velocity plot of the active seismic survey performed in Lucerne (Switzerland). The elaborations for the vertical component using the Fourier-based beamforming along the whole trace record (a) and using the wavelet $t-f-k(\mathbf{b})$ are presented. The array consisted in a linear configuration of 12 seismological stations with $4 \mathrm{~m}$ interdistance. Five shots were recorded continuously, in this example with a shot-offset of $2 \mathrm{~m}$. The lower and the higher resolution limits for this array geometry are indicated with red lines. Automatic mode picking is presented in green dots, while manual mode interpretation is in black solid line

dispersion curve obtained from a passive acquisition survey performed at the same location. This example shows, therefore, the advantages of combining active seismic with ambient noise processing to compensate for the respective limitations of the two methods.

To decompose the wave field, a time-frequencywavenumber analysis based on the wavelet-transform has been applied to the active seismic records. To compute the continuous wavelet transform we propose the use of a simple alternative mother wavelet. The wavelet is similar to the Morlet wavelet and produces comparable results for the same values of the nondimensional frequency. However, with respect to the classical Morlet base function, the proposed wavelet is stable and satisfies the admissibility conditions for values of $C_{0}$ smaller than 6 , a 

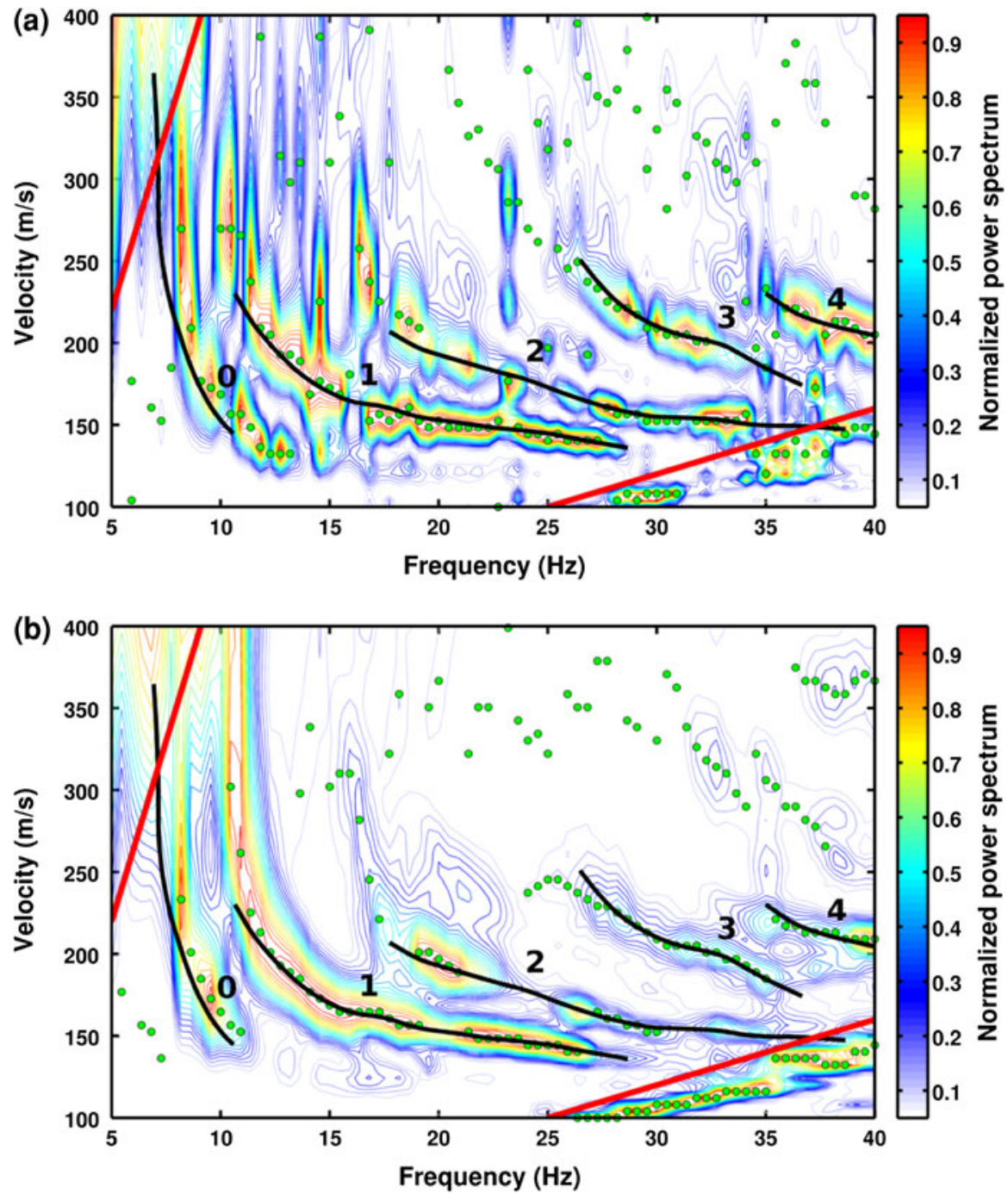

Figure 15

Same as Fig. 14, but for the radial direction of motion. The identified Rayleigh wave modes are consistent between the two processed components (in black the curves obtain from the vertical component, for comparison), but they clearly show a different energy content

condition that ensures the achievement of a higher theoretical temporal resolution.

As a main disadvantage, the continuous recordings we use are only possible with highly specialized equipment, whose rather high cost limits widespread use in the geophysics community. Nevertheless, we would like to stress that new types of seismologicallike hybrid devices are nowadays under development (e.g., Picozzi et al., 2010), and begin to be available on the market at relatively low prices (e.g., http://www. tromino.eu/), if compared to standard seismic stations. This new trend might be advantageous in supporting the development of nonconventional seismic processing techniques.

As an additional disadvantage, moreover, the presented approach is computationally more expensive when compared to previous $f-k$ methods, since it requires the recomputations of the covariance matrix for all permutations of the analyzed frequencies, phase velocities and source delay times. Furthermore, such a procedure has to be repeated for all recorded shots before stacking. Nevertheless all the search parameters are here independent, and thus the algorithm is easily parallelizable and scalable. It is well 


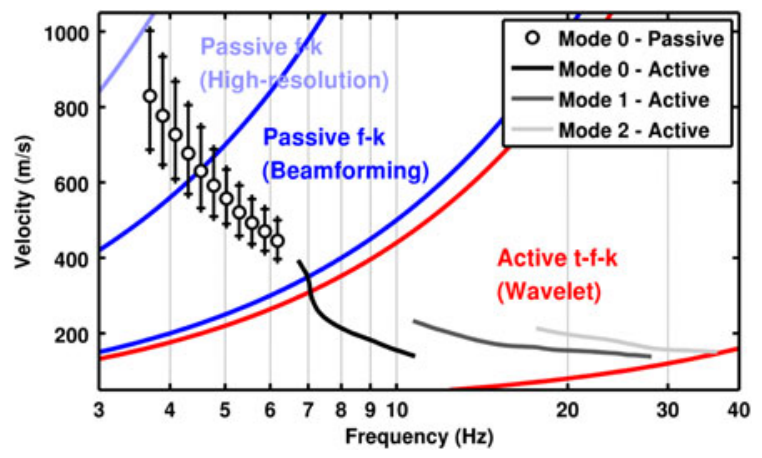

Figure 16

Comparison between Rayleigh wave dispersion curves obtained with active and passive seismic techniques. Resolution bounds of the different methods are presented. The velocity jump in the fundamental mode is most likely caused by the presence of a large seismic impedance contrast at about $10 \mathrm{~m}$

suited for distributed computing on multi-core/multiprocessor machines.

With few modifications, we are confident that the presented method might also be successfully extended to ambient noise processing, to enhance the capability of separating out the different wave contributions (e.g., body and surface waves). As a future development, we plan to extract and analyze the surface wave amplitude information from the $t-f-k$ power spectrum estimates. On three-component recordings, for example, this might allow the estimation of the Rayleigh wave ellipticity function in active seismic experiments. We plan, moreover, to implement and to exhaustively test a high resolution version of the method, which is nowadays necessary to compete with existing standard high resolution techniques for surface wave analysis.

\section{Acknowledgments}

This research was supported by the Swiss National Science Foundation within the project HAZARD 2010 (SNF No. 200021-112284 and No. 200020126690) and by ENSI (Swiss Federal Nuclear Safety Inspectorate). We would like to thank Sabine Latzel and Juerg Schuler for providing the synthetic datasets which have been used to validate the method. A special thank goes also to Prof. Stewart Greenhalgh for all his precious advise.

\section{REFERENCES}

K. AKI and P.-G. Richards. Quantitative seismology. Theory and methods. W.H. Freeman and Company, New York, 1980.

J.-P Burg. Maximum entropy spectral analysis. 37th Annual International SEG Meeting, Oklahoma City, 1967.

J. CAPON. High resolution frequency wavenumber spectrum analysis. Proc. IEEE, 57:1408-1418, 1969.

G. Dal Moro, E. Forte, M. Pipan, and M. Sugan. Velocity spectra and seismic signal identication for surface-wave analysis. Near Surface Geophysics, 4:243-251, 2006.

I. DAUBECHIES. The wavelet transform, time-frequency localization and signal analysis. IEEE Transaction on Information Theory, 36, 1990.

M. FARGE. Wavelet transforms and their applications to turbulence. Ann. Rev. Fluid Mech., 24:395-457, 1992.

W. Friederich and J. Dalkolmo. Complete synthetic seismograms for a spherically symmetric earth by a numerical computation of the greens function in the frequency domain. Geophys. J. Int., 122:537-550, 1995.

P. Goupillaud, A. Grossman, and J. Morlet. Cycle-octave and related transforms in seismic signal analysis. Geoexploration, 23:85-102, 1984.

M. Holschneider, M. S. Diallo, M. Kulesh, M. Ohrnberger, E. Lueck, F. Scherbaum, Characterization of dispersive surface waves using continuous wavelet transforms, Geophys. Jour. Int. 163:463-478, 2005.

M. HORIKE. Inversion of phase velocity of long-period microtremors to the s-wave velocity structure down to the basement in urbanized areas. J. Phys. Earth, 33:59-96, 1985.

D.-S KIM and H.-C PARK. Determination of Dispersion Phase Velocities for SASW method Using Harmonic Wavelet Transform, Soil Dynamics and Earthquake, 2:675-684, 2002.

A. Kritski, D. A. Yuen, A. P. Vincent, Properties of near surface marine sediments from wavelet correlation analysis, Geophysical research letters, 29:1922-1925., 2002.

M. Kulesh, M. Holschneider, M. Ohrnberger, and E. Lück: Modeling of wave dispersion using continuous wavelet transforms II: wavelet based frequency-velocity analysis, Pure and Applied Geophysics., 165:255-270, 2008.

J.-N. Loule, Faster, better: shear-wave velocity to 100 meters depth from refraction microtremor arrays, Bull. Seism. Soc. Am., 91:347-364, 2001.

R.-T. Lacoss, E.-J. Kelly, and M.-N. ToKsz. Estimation of seismic noise structure using arrays. Geophysics, 34:21-38, 1969.

Y., Luo, J., XIA., R.-D., Miller, Y., Xu, J., Liu, and Q., Liu, Rayleigh-wave dispersive energy imaging by high-resolution linear Radon transform: Pure and Applied Geophysics, 165(5), 903-922, 2008.

G.-A. McMechan and M.-J. Yeduin. Analysis of dispersive wave by wavefield transformation. Geophysics, 46:869-874, 1981.

G. Nolet and G.-F. PAnZA. Array analysis of seismic surface waves: limits and possibilities. Pure and Applied geophysics, 114:776-790, 1976.

C.-B. Park, R.-D. Miller, and J. XIA. Multichannel analysis of surface waves, Geophysics, 64:800-808, 1999.

C.-B. Park, R.-D. Miller, N. Ryden, J. XiA, and J. Ivanov. Combined use of active and passive surface waves. Journal of Environmental and Engineering Geophysics, 10, 3:323-334, 2005. 
M. Picozzi, C. Milkereit, S. Parolai, K.-H. Jaeckel, I. Veit, J. Fischer, J. Zschau, GFZ Wireless Seismic Array (GFZ-WISE), a Wireless Mesh Network of Seismic Sensors: New Perspectives for Seismic Noise Array Investigations and Site Monitoring Sensors, 10:3280-3304, 2010.

V. PoGGI and D. FäH. Estimating Rayleigh wave particle motion from three-component array analysis of ambient vibrations. Geophys. J. Int., 180:251-267, 2010.

V. Poggi, D. Fäh, J. BuRJanek, and D. Giardini. The use of Rayleigh wave ellipticity for site-specific hazard assessment and microzonation. Application to the city of Lucerne, Switzerland. Geophys. J. Int., 188, 3:1154-1172, 2012.

R.-O. Schmidt. Multiple emitter location and signal parameter estimation. IEEE Trans. Antennas Propagation, 34:276-280, 1986.

J. SCHULER. Joint inversion of surface waves and refracted P- and S-waves. Master Thesis, Swiss Federal Institute of Technology (ETH), Zurich, 2008.

N.-M. Shapiro, M. Campillo, L. Stehly, and M.-H. Ritzwoller. High-resolution surface wave tomography from ambient seismic noise. Science, 307, 5715:1615-1618, 2005.

Y. SHENG. Wavelet transform, in "The transforms and applications handbook". A.D. Poulariskas Ed. Chap. 10, CRC and IEEE Press, Boca Raton, 1995.
K.-H. Stokoe and S. Nazarian. Use of Rayleigh wave in liquefaction studies. Proc. Of the "measurement and use of shear wave velocity for evaluating dynamic soil properties", ASCE, N.Y., 1-17, 1985.

C. Strobbia. Surface wave method: acquisition, processing and inversion. PhD thesis, Politecnico di Torino, 2003.

K. Tокімatsu. Geotechnical site characterization using surface waves. In Proc. 1st Intl. Conf. Earthquake Geotechnical Engineering, 1333-1368, 1997.

M. Wathelet, D. Jongmans, M. Ohrnberger, and S. BonnefoyClaudet. Array performances for ambient vibrations on a shallow structure and consequences over vs inversion. Journal of Seismology, 12, 1:1-19, 2008.

J. XIA, R.-D. MilLER, and C.-B. PARK. Estimation of near-surface shear-wave velocity by inversion of Rayleigh waves. Geophysics, 64:691-700, 1999.

J. XIA, Y. Xu, and R.-D MiLLER. Generating an image of dispersive energy by frequency decomposition and slant stacking. Pure and Applied Geophysics, 164:941-956, 2007.

Ö., Yilmaz, Seismic data processing, Society of Exploration Geophysicists, Tulsa, OK. 1987. 